\title{
Joint Determination of Slip and Stress Drop in a Bayesian Inversion Approach: A Case Study for the 2010 M8.8 Maule Earthquake
}

\author{
Lifeng Wang, ${ }^{1}$ Gert ZÖller, ${ }^{2}$ and Sebastian Hainzl ${ }^{3}$
}

\begin{abstract}
Stress drop is a key factor in earthquake mechanics and engineering seismology. However, stress drop calculations based on fault slip can be significantly biased, particularly due to subjectively determined smoothing conditions in the traditional least-square slip inversion. In this study, we introduce a mechanically constrained Bayesian approach to simultaneously invert for fault slip and stress drop based on geodetic measurements. A Gaussian distribution for stress drop is implemented in the inversion as a prior. We have done several synthetic tests to evaluate the stability and reliability of the inversion approach, considering different fault discretization, fault geometries, utilized datasets, and variability of the slip direction, respectively. We finally apply the approach to the 2010 M8.8 Maule earthquake and invert for the coseismic slip and stress drop simultaneously. Two fault geometries from the literature are tested. Our results indicate that the derived slip models based on both fault geometries are similar, showing major slip north of the hypocenter and relatively weak slip in the south, as indicated in the slip models of other studies. The derived mean stress drop is 5-6 MPa, which is close to the stress drop of $\sim 7 \mathrm{MPa}$ that was independently determined according to force balance in this region LutTRELl et al. (J Geophys Res, 2011). These findings indicate that stress drop values can be consistently extracted from geodetic data.
\end{abstract}

Key words: Stress drop, Fault slip, Bayesian, Geodetic measurements.

\section{Introduction}

Stress drop is a fundamental quantity for understanding fault mechanics of earthquakes, and is important in engineering seismology and seismic

Electronic supplementary material The online version of this article (doi:10.1007/s00024-014-0868-x) contains supplementary material, which is available to authorized users.

1 China Earthquake Networks Center, Beijing, China. Email: chsxwlf@hotmail.com; wanglifeng@seis.ac.cn

2 University of Potsdam, Potsdam, Germany.

3 GFZ German Research Centre for Geosciences, Potsdam, Germany. hazard. The rapid drop of shear stress during an earthquake controls the seismic radiation from the source and thus the ground motion (e.g., HANKs and McGuire, 1981). Therefore, reliable estimates of earthquake stress drop are important for calculating seismic shaking and analyzing long-term seismic hazards, such as those related to the recurrence interval of large earthquakes (e.g., FukaO and FuRUMoto, 1978; Parsons, 2006).

There are several ways to estimate the stress drop, including (1) estimations based on geologically observed average fault slip, characteristic source dimension, and the shear modulus of the host rock (KANAMORI and ANDERson, 1975), (2) estimation based on the corner frequency or source duration from seismic signals with the condition that the finite rupture can be represented by an equivalent point source (BRUNE, 1970; MADARIAGA, 1976), and (3) estimation based on slip distribution on the fault plane (e.g., Hartzell and Helmberger, 1982; RiPPERGER and MAI, 2004; Bouchon et al., 1998; AndREws, 1974). The first approach based on geological data usually involves large uncertainties, because it only utilizes average slip and simple stress change models of a circular/rectangular dislocation. The second approach based on the source spectrum also leads to significant errors, because the large earthquakes (especially with complex rupture) cannot be approximated by a point-source, and thus the relationship between high-frequency ground motion and static source characteristics is ambiguous (AtKinson and Beresnev, 1997). Even if the point source is an appropriate approximation, the small uncertainty in the corner frequency derived from the source spectrum may lead to a large uncertainty in the stress drop due to their cubic relation (BRUNE, 1970). As a consequence, most published stress drop values, 
even for the same earthquakes, exhibit large epistemic errors (CоттоN et al., 2013).

For large earthquakes (ruptures), it is convenient to estimate the stress drop based on the inverted slip distribution by calculating the shear stress change on the fault plane (MAI and BEROZA, 2000), given that geodetic and seismological measurements are available. However, non-uniqueness of fault slip inversion is well-known (e.g., Snieder and Trampert, 1999), due to usually limited observations, different fault parameterizations (e.g., PAGE et al., 2009), different algorithms (e.g., Du et al., 1992), and different considerations of the measurement uncertainties (e.g., Lohman and Simons, 2005), etc. In particular, the subjectively applied smoothing condition can greatly bias the inverted fault slip (TARANTOLA, 2005) and thus, the estimation of stress drop (see Sect. 5.1).

Nevertheless, the multiplicity of solutions in slip inversion can be conveniently expressed using a Bayesian approach, which has been applied in several studies (e.g., Yabuki and Matsu'ura, 1992; Fukuda and Johnson, 2008; Monelli and MaI, 2008; Monelli et al., 2009; SimOns et al., 2011), where the inversion problem is formulated only from the kinematic point of view. Recently, some mechanical constraints have been incorporated into fault slip inversion. For example, WANG et al. (2012) considered stress coupling between the mainshock and postseismic relaxation process and applied postseismic displacements as additional constraints to invert for the coseismic rupture. Sun et al. (2011) simply utilized a uniform stress drop in the rupture area as a constraint to relax the traditionally applied smoothing condition. In this study, we consider the heterogeneity of slip due to roughness on the fault plane, and consider the stress drop on the fault plane as a spatially random field. Random heterogeneity and related effects have been widely applied in dynamic modeling (e.g., Andrews, 1980; Mai and Beroza, 2002; Guatteri et al., 2004). The stress drop of an earthquake, the difference between the stress across a fault before and after the occurrence of the earthquake, is considered here to be Gaussian distributed, by assuming that the stress drops in the discretized rupture zone are random numbers around a central value, which characterizes the stress drop of the earthquake. The Gaussian distribution is frequently utilized for many situations as a first-order description because it represents the complete randomness when the mean and variance are given; further details about our selection of the Gaussian distribution are discussed in Sect. 5.1.

As a case study, we apply the approach to the 2010 M8.8 Maule earthquake. This earthquake was not an unexpected event. It occurred in a 'seismic gap' of the subduction zone between the Nazca and South America plates (Ruegg et al., 2002, 2009; Moreno et al., 2008). Therefore, this region has long been monitored. For the M8.8 Maule earthquake, several coseismic slip models (LAY et al., 2010; LoRITO et al., 2011; VignY et al., 2011; PollitZ et al., 2011; Tong et al., 2010; Moreno et al., 2012) have been published, which were all derived from the least-square (LS) method. In this study, we revisit this earthquake and utilize a Bayesian approach that incorporates physical constraints to simultaneously obtain the full solutions for both fault slip and stress drop.

We use the coseismic displacement data published in several geodetic studies. The GPS data are compiled from VignY et al. (2011) and Moreno et al. (2012), all of which are processed in a fixed South American reference frame. Meanwhile, Tong et al. (2010) have made the assembled InSAR data available. The dense coseismic displacement measurements provide a good opportunity to test our inversion approach.

\section{Inversion for Stress Drop and Fault Slip in Bayesian Approach}

The Bayesian approach provides a mathematical framework that allows for a rigorous treatment of the model uncertainties and provides a full solution space of the unknown parameters. For a given fault geometry, we assume a Gaussian distribution of the error $\mathbf{e}$ (Beck and Katafygiotis, 1998; Beck, 2010; TarantOLA, 2005) stemming only from the measurement uncertainty in the geodetic data as a simplification, while ignoring the usually unknown uncertainties in the forward model related to fault geometry and crustal properties. Then, the likelihood function that is the conditional density of observations $\left(\mathbf{y}_{n} \times 1\right)$ given slip $\mathbf{b}_{M \times 1}$ is 


$$
\begin{aligned}
& p(\mathbf{y} \mid \mathbf{b})=(2 \pi)^{-n / 2}|\Sigma|^{-1 / 2} \\
& \exp \left[-\frac{1}{2}(\mathbf{y}-\mathbf{G b})^{T} \Sigma^{-1}(\mathbf{y}-\mathbf{G b})\right]
\end{aligned}
$$

where $\mathbf{G}$ (with dimension of $n \times M$ ) is the matrix of Green's functions with $n$ being the number of observations and $M$ being the number of fault slip. If we fix the slip is along the rake direction, $M$ has the same number as the fault patches. In the case of slip direction being not fixed, $M$ is two times the number of fault patches. $\Sigma_{n \times n}$ is a diagonal matrix of the variances of the measurements. Thus, the observation data with relatively large uncertainties have less constraint in the inversion than those with smaller uncertainties.

In this study, instead of using Laplacian smoothing (e.g., WANG et al., 2012) of the slip on the fault plane, we incorporate a mechanical constraint into the model. Since static stress drop is the measure of the overall reduction in shear stress due to slip on the fault zone (RuFF, 1999), we simply consider the stress drop as the decrease of shear stress produced by the earthquake. Given that the rupture area is usually large for a big earthquake and involves a large number of fault patches, we assume that the stress drops (on the fault patches) in the discretized rupture area are the uncorrelated random numbers around a central value, the stress drop of the earthquake. In particular, we assume a Gaussian distribution with mean $\tau_{0}$ and variance $\alpha^{2}$, i.e., $\mathbf{S b} \sim \boldsymbol{N}\left(\tau_{0}, \alpha^{2} \mathbf{I}\right)$ for fault patches $i(i \in 1 \ldots M)$ with negative shear stress change, i.e. $[\mathbf{S b}]_{i}<0$. Here, $\mathbf{S}_{M} \times{ }_{M}$ is the matrix that projects the slip to shear stress using Okada's solution (OKADA, 1992). Additionally, considering only the non-negative slip to preserve consistent rupture direction during the earthquake, the prior probability density (PDF) of $\mathbf{b}$ given $\alpha^{2}$ and $\tau_{0}$ is,

$$
p\left(\mathbf{b} \mid \tau_{0}, \alpha^{2}\right) \sim \begin{cases}\left(2 \pi \alpha^{2}\right)^{-M / 2} \mathrm{e}^{\frac{-\sum_{i=1}^{M} H\left(-\left\langle\mathbf{S b}_{i}\right\rangle\left\langle\left(\mathbf{s b}_{i} i-\tau_{0}\right)^{2}\right.\right.}{2 \alpha^{2}}} & \mathbf{b}_{\max } \geq \mathbf{b} \geq \mathbf{0}, \\ 0 & \text { otherwise }\end{cases}
$$

where $\mathbf{b}_{\max } \geq \mathbf{b} \geq \mathbf{0}$ means $\mathrm{b}_{\max } \geq[\mathbf{b}]_{i} \geq 0$ for $i=1 \ldots M$, with $\mathrm{b}_{\max }$ being the upper limit of slip value (we set $b_{\max }=30 \mathrm{~m}$ for the Maule earthquake). $H(x)$ is the Heaviside function $(H(x)=1$ for $x>0$; else $H(x)=0$ ). Since we are mainly interested in the description of the rupture area, we only considered here negative stress (i.e., stress drop) values. Nevertheless, this prior information (Eq. 2) also serves as a weak constraint to the area where shear stress is positive, because any increase in the slip of such area could change the local stress from a positive to a negative value so that it contributes to the prior distribution.

In our application to the Maule earthquake, we fix the rake angle to $110^{\circ}$ according to the focal mechanism of the Maule earthquake (Lorito et al., 2011; VIGNY et al., 2011) and the orientation of the convergence between the Nazca and South America plates. The dislocation along a rake angle of $110^{\circ}$ dominates the coseismic rupture of the Maule earthquake.

In this slip inversion with the mechanical constraint, $\tau_{0}$ provides the stress drop of the earthquake reflected from geodetic measurements. Since we have no specific knowledge about $\tau_{0}$, we specify a uniform PDF between 0.1 and $20 \mathrm{MPa}$ (with $\tau_{0}$ being negative values in the inversion), which covers the usually measured average stress drop of 0.1-10 MPa (KANAMORI and ANDERSON, 1975; Kanamori, 1994) or 0.2-20 MPa (Shearer et al., 2006) for individual events. The variance $\alpha^{2}$ value is related to the heterogeneity of stress on the fault plane; in particular, larger $\alpha^{2}$ values correspond to higher levels of heterogeneity. We apply a uniform PDF between 0.1 and $20 \mathrm{MPa}^{2} \mathrm{PDF}$ for $\alpha^{2}$, the same range as for $\tau_{0}$.

According to Bayes' theorem (BAYES and Price, 1763; Daston, 1988; Косн, 2007), the posterior is the product of the likelihood function and the prior PDFs, and thus

$$
p\left(\mathbf{b}, \tau_{0}, \alpha^{2} \mid \mathbf{y}\right) \sim \begin{cases}p\left(\mathbf{b} \mid \tau_{0}, \alpha^{2}\right) p(\mathbf{y} \mid \mathbf{b}) & 20 \geq \tau_{0}, \alpha^{2} \geq 0.1 ; \mathbf{b} \geq \mathbf{0} \\ 0 & \text { otherwise }\end{cases}
$$

According to the (unnormalized) posterior PDF of Eq. 3, we draw the samples of $\mathbf{X}=\left\{\mathbf{b}^{T}, \tau_{0}, \alpha^{2}\right\}_{1 \times(M+2)}$ using the Markov Chain Monte Carlo (MCMC) method (Gelfand and Smith, 1990; Metropolis et al., 1953; HAstings, 1970). Briefly, the MCMC method operates in the following way: firstly, the procedure starts from the initial state $\mathbf{X}_{0}$ with its unnormalized posterior 

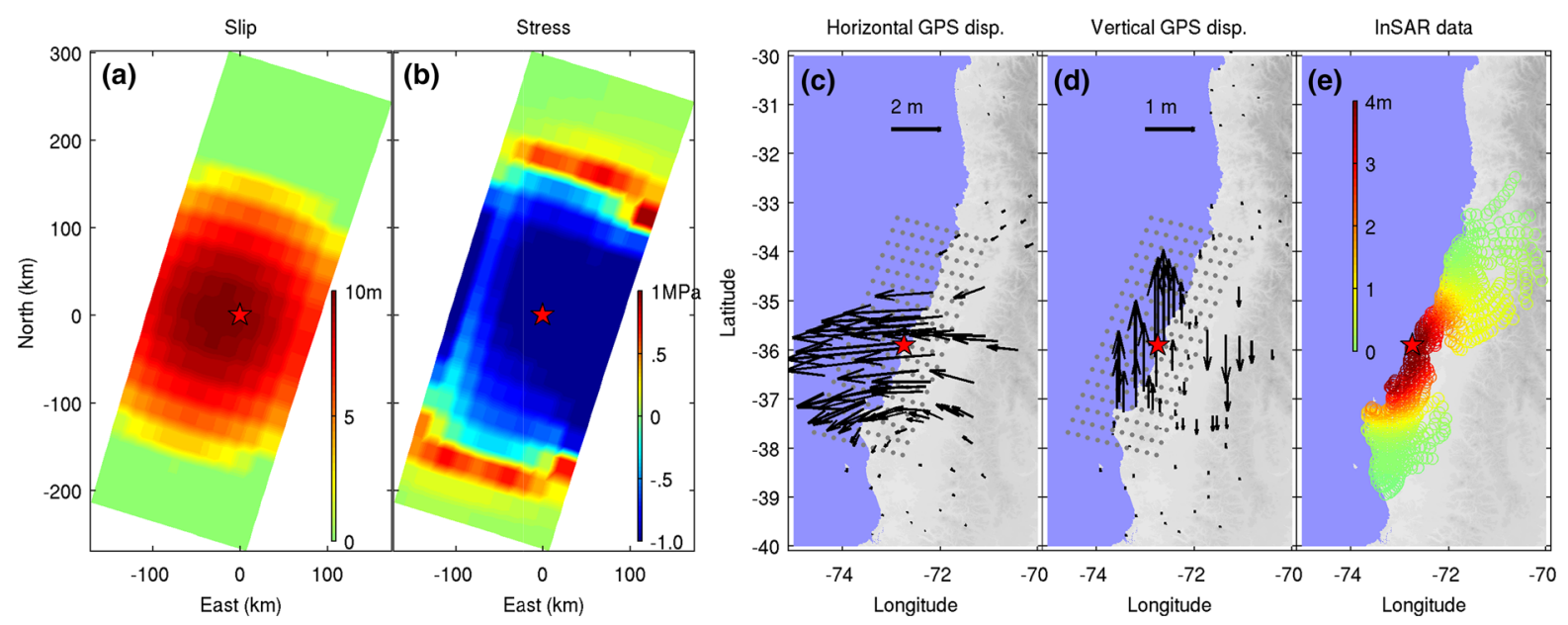

Figure 1

Color-coded synthetic input slip model (a), the corresponding shear stress changes along the rake angle of $110^{\circ}$ (b) and the surface displacements (plots $\mathbf{c}-\mathbf{e}$ ). The star marks the hypocenter of the Maule earthquake. The dotted rectangle in panel (c) and (d) indicates the location of the fault plane

distribution $p\left(\mathbf{X}_{0} \mid \mathbf{y}\right)$. Secondly, the new trial value $\mathbf{X}^{\prime}$ is randomly generated (based on a uniform distribution) in space $\left(\mathbf{X}_{0}-\Delta \mathbf{X}, \mathbf{X}_{0}+\Delta \mathbf{X}\right)$, with $\Delta \mathbf{X}$ being the step size of the Markov Chain, and selected as the next state that $\mathbf{X}_{1}=\mathbf{X}^{\prime}$ if $\min \left(1, \frac{p\left(\mathbf{X}^{\prime} \mid \mathbf{y}\right)}{p\left(\mathbf{X}_{0} \mid \mathbf{y}\right)}\right) \geq u$, where $u$ is a random number generated from a uniform distribution over the interval $[0,1]$. In the same way, the states $\mathbf{X}_{2}, \mathbf{X}_{3} \ldots$ are subsequently generated and selected. After a large number of steps, the procedure converges to the probability density $p(\mathbf{X} \mid \mathbf{y})$ for any initial state, and the samples drawn can approximate the posterior PDF. In the simulation procedure, $\sim 5 \times 10^{8}$ iterations in total are performed. We neglect the first $2 \times 10^{7}$ samples drawn, and then take one out of each 1,000 samples drawn to construct the posterior PDF. The selected optimal value of each parameter has the maximum value of the marginal posterior PDF, and the uncertainty is evaluated by the maximum/minimum value after dropping $2.5 \%$ of the samples at each side of the distribution according to WANG et al. $(2012,2014)$. A similar approach has also been applied by Minson et al. (2013).

\section{Synthetic Test to the Inversion Approach}

We first calibrate the inversion approach by a series of synthetic tests. Utilizing the fault geometry of the
Maule earthquake provided by USGS (http://earthquake. usgs.gov/), we simulate a synthetic slip along $110^{\circ}$ and centered at the hypocenter of the Maule earthquake, where the slip has an assumed peak value of $10 \mathrm{~m}$. The amount of the synthetic slip decays with the distance to the hypocenter following a parabolic function (JOHNSON, 1979). The fault plane is discretized into $18 \times 10$ rectangular patches, each with dimension of $30 \times 20 \mathrm{~km}$.

Figure 1 displays the input slip model, its shear stress change along the rake direction $\left(110^{\circ}\right)$, and the produced surface displacements on the sites where the GPS/InSAR measurements are available for the Maule earthquake. The synthetic displacements include random noise simulated by Gaussian distributions with zero mean and standard deviation of $3 \mathrm{~mm}$ for horizontal GPS measurements, $10 \mathrm{~mm}$ for vertical GPS data and InSAR, respectively.

Utilizing the synthetic displacements and the fault parameterization, we draw samples according to the (unnormalized) posterior PDF of Eq. 3 based on Markov Chain Monte Carlo sampling, and select the optimal solution according to the maximum value of the marginal PDF that is constructed from the drawn samples. The sampling process (including $\sim 5 \times 10^{8}$ samples) based on the MCMC method is shown in Fig. 2a, where the logarithmic values of the sampled (unnormalized) posterior PDF of Eq. 3 (labeled as ' $\log \mathrm{P}$ ') are plotted against the iteration number. 

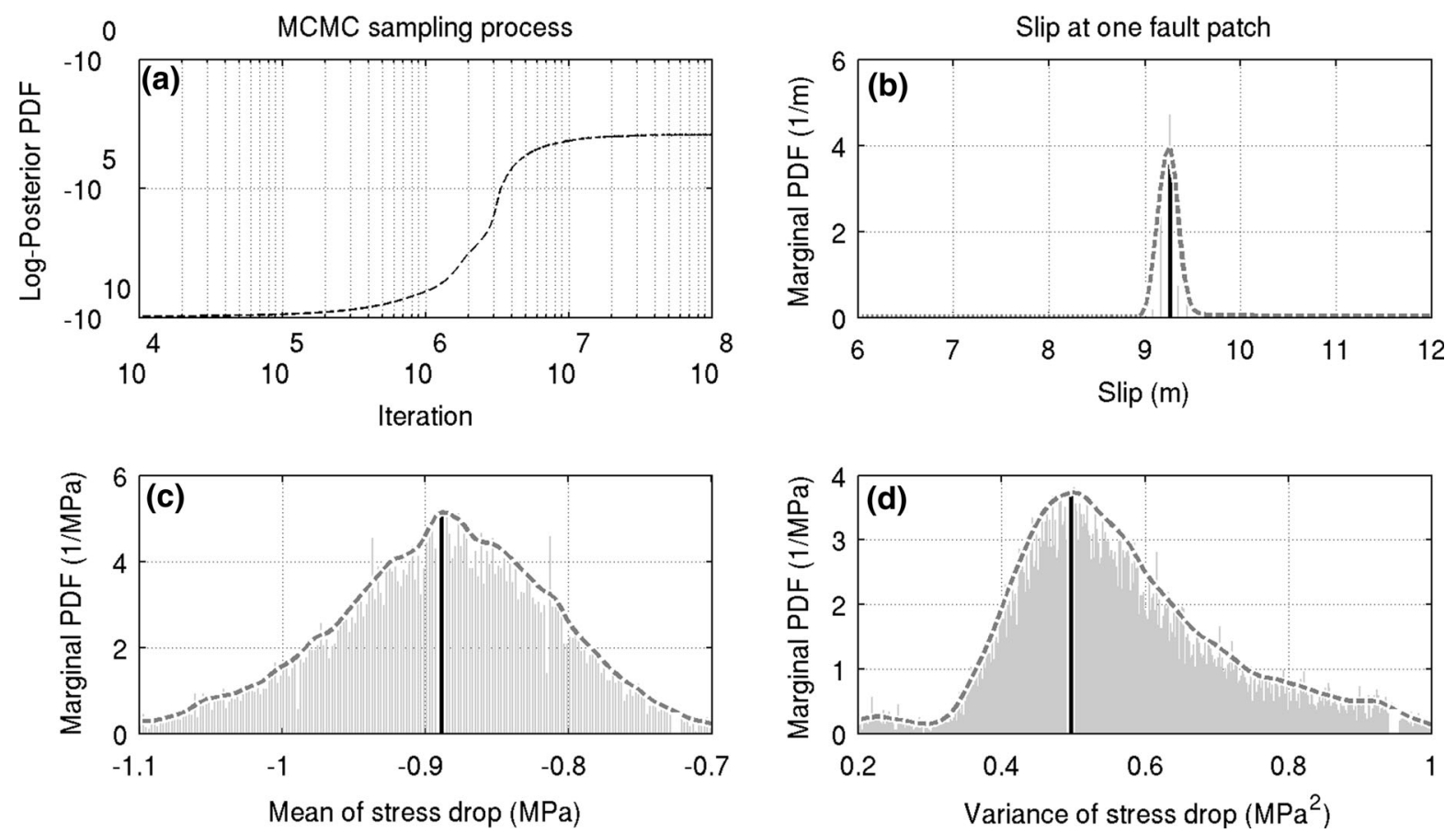

Figure 2

Sampling results for Test-0. a The logarithmic values of the sampled posterior PDF of Eq. 3 are plotted against the iteration number during the MCMC sampling process; marginal posterior probability density (abbreviated as 'Marginal PDF') constructed from the MCMC generated samples for slip at a location marked with ' + ' in Fig. 3a, with input slip value of $9.36 \mathrm{~m}$ (b), for mean stress drop of the event (c) and the variance of stress drop (d). In panels b-d, the black line marks the optimal value determined according to the maximum based on the marginal PDF

Figure 2a shows that the procedure approaches the maximum probability density during the MCMC sampling. We construct the marginal PDF for each unknown parameter based on the samples drawn. The resulting marginal PDFs for slip at one fault location and for stress drop (mean and variance) are displayed in Fig. 2b-d. All of them show single peaked distributions. Thus, we may reliably select the optimal parameters that have the highest probability densities as representative values. We show the optimal solutions for the slip and stress drop in Fig. 3, where the errors of the slip residuals (the differences from the synthetic slip of Fig. 1a) are shown in Fig. 3a and the uncertainties of the slip estimates are provided in Fig. $3 b$. The uncertainties are evaluated according to the $95 \%$ credibility interval and are calculated by the difference between the optimal value and the maximum value of the samples drawn after neglecting $5 \%$ of the samples with the lowest probability density (WANG et al., 2012). The results display that the optimal solution of slip is close to the input model with variance reductions (BECKER et al., 2005) in both slip $\left(\mathrm{VR}_{\mathrm{slp}}\right)$ and surface displacements $\left(\mathrm{VR}_{\mathrm{dis}}\right)$ higher than $99 \%$, where $\mathrm{VR}_{\text {slp }}$ is evaluated based on the difference of the derived optimal solutions from the synthetic slip model shown in Fig. 1a; while $\mathrm{VR}_{\mathrm{dis}}$ is based on the difference between the estimated and synthetic displacements. Moreover, the slip error is indicated to be in the range of estimation uncertainty. The surface displacements produced by the optimal slip model are provided in the supplementary material (Fig. S1).

The probability density of the inverted stress drop with mean value of $-0.87 \mathrm{MPa}$ and standard deviation of $0.72 \mathrm{MPa}$ (variance of $0.52 \mathrm{MPa}^{2}$ ) is shown in Fig. 3c, where the histogram of true stress drops (for the different fault patches of the input slip model of Fig. 1a) is displayed by gray bars, with a mean of $0.93 \mathrm{MPa}$ and standard deviation of $0.53 \mathrm{MPa}$. Although the estimated probability density somewhat differs from the real stress drop distributions likely due to the relatively small number of fault patches, 

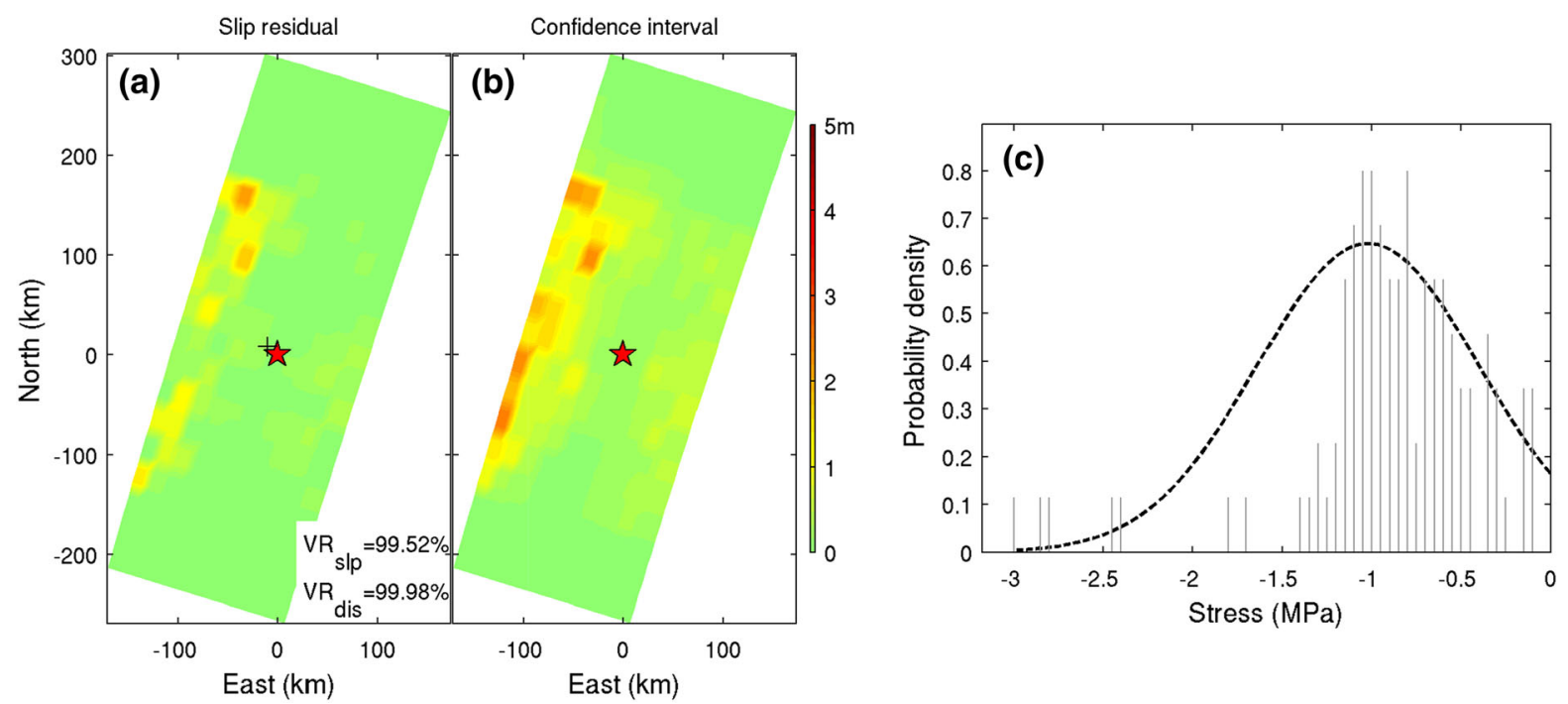

Figure 3

Inversion results based on the synthetic displacements shown in Fig. 1. a color-coded residual slip (the difference between the synthetic and estimated slip); b uncertainty of the slip estimation in terms of the $95 \%$ credibility interval; c probability density distribution of the estimated stress drop (dashed curve) and the histogram plot (gray bars) of the synthetic stress drop on the fault patches that is normalized by the total number of the patches. In panel (a), '+' marks the patch on which the sampled slip values are presented in Fig. 2b

the estimation broadly outlines the true values. The dominant stress drop values are located in the peak area of the estimated probability density, indicating that the inversion approach is able to reconstruct the general statistical features of the stress drop field. This inversion test is labeled as 'Test 0 '.

In order to investigate the stability (especially with respect to the stress drop estimation) of the inversion approach depending on fault discretization, fault geometry, size of datasets and possible bias in the rake angle, we perform additional tests. First, we test two different discretizations for the USGS fault plane, $15 \times 6$ patches with size of $35 \times 32 \mathrm{~km}$ (labeled as Test I) and $26 \times 11$ patches with size of $21 \times 17 \mathrm{~km}$ (Test II). Second, we use Lorito's fault plane (LoRITo et al., 2011), which is discretized as $25 \times 8$ patches with size of $25 \times 25 \mathrm{~km}$ (Test III). Third, instead of using the whole synthetic data in Test-0, we do the inversion based on either GPS data (Test IV) or InSAR data (Test V). Fourth, we test the stability of the inversion considering the effect of possible error in the rake angle. Assuming a systematic error of $3^{\circ}$, we invert for fault slip and stress drop along the rake angle of $113^{\circ}$ (Test-VI). Furthermore, considering the heterogeneity in the rake angle, we use the fault slip along the rake angle with mean of $110^{\circ}$ and standard deviation of $3^{\circ}$ to simulate the surface displacements, based on which we invert for the slip along the fixed rake angle of $110^{\circ}$ (TestVII). In this case, the true stress drop distribution (negative shear stress along the rake angle of $110^{\circ}$ ) is slightly different, with a mean of $-0.90 \mathrm{MPa}$ and variance of $0.56 \mathrm{MPa}^{2}$. In the Tests IV-VII, the fault parameterizations are the same as that in the Test- 0 .

With $\mathrm{VR}_{\mathrm{dis}}$ values higher than $99 \%$, our results indicate that the slip models obtained can explain the surface displacements well in all of the seven synthetic tests. Because of the differences in the fault parameterizations, it is not easy to fairly compare variance reduction in slip $\left(\mathrm{VR}_{\text {slp }}\right)$ for Tests I-III (different patch size) and VI-VII (different slip direction). However, the optimal slip models obtained, shown in the supplementary material (Figs. S2-8), indicate that the fault slip is well-retrieved. We display the stress drop estimates in Fig. 4. Depending on the model setups or the utilized datasets, the resulting stress drop values differ. For example, stress drop derived based on Lorito's fault plane is largely biased from the real values (gray color bars in Fig. 4) or the estimates of Test-0 (the 


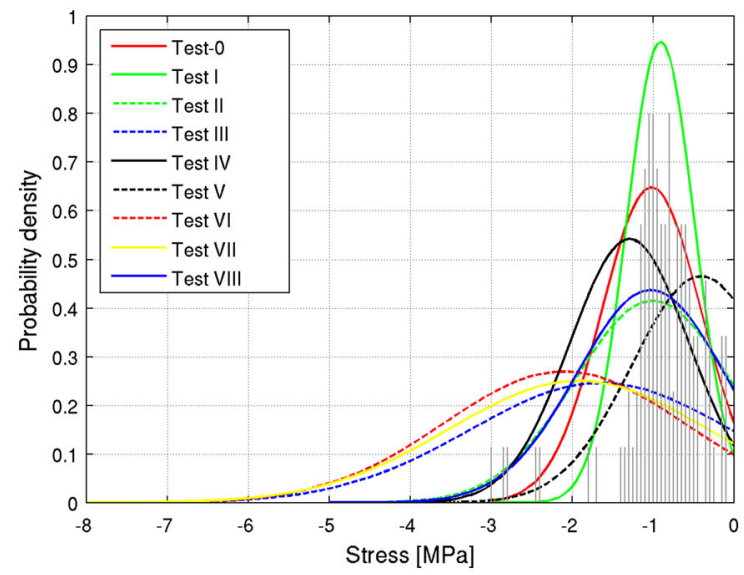

Figure 4

Estimated probability density functions of stress drop for Tests 0VIII. The true stress drop values at the slip patches of the synthetic model are shown by gray bars

red curve in Fig. 4). This is related to the obvious difference between USGS' and Lorito's fault geometry. Furthermore, if the rake angle is erroneously set, the estimated mean stress drop also deviates from the true value (Test-VI and Test-VII). Nevertheless, the differences are small $(<1.5 \mathrm{MPa})$ and thus the main statistical features are preserved. Stress drop derived in the other four tests (I, II, IV and V) displays mean values of $\sim-1 \mathrm{MPa}$, close to the dominant values of true stress drops. On the other hand, the standard deviation of stress drop is sensitive to patch size in the fault discretization. Generally speaking, the model with larger patch size provides a narrower statistical distribution for stress drop and smaller standard deviation, which is reasonable because small heterogeneity cannot be resolved when large fault patches are used in the inversion. While the fault discretization in the model only has an impact on the estimation of the heterogeneity (characterized by $\alpha^{2}$ ), the biased fault geometry and rake angle may lead to a relatively large deviation in both mean and standard deviation of stress drop (e.g., Tests III, VI and VII). These synthetic tests again reflect the non-uniqueness of the inversion results depending on the data utilized, fault geometry, and fault parameterization, etc.

Further, we perform a test based on the synthetic displacements produced by a slip cluster with random noise in order to simulate heterogeneity in slip
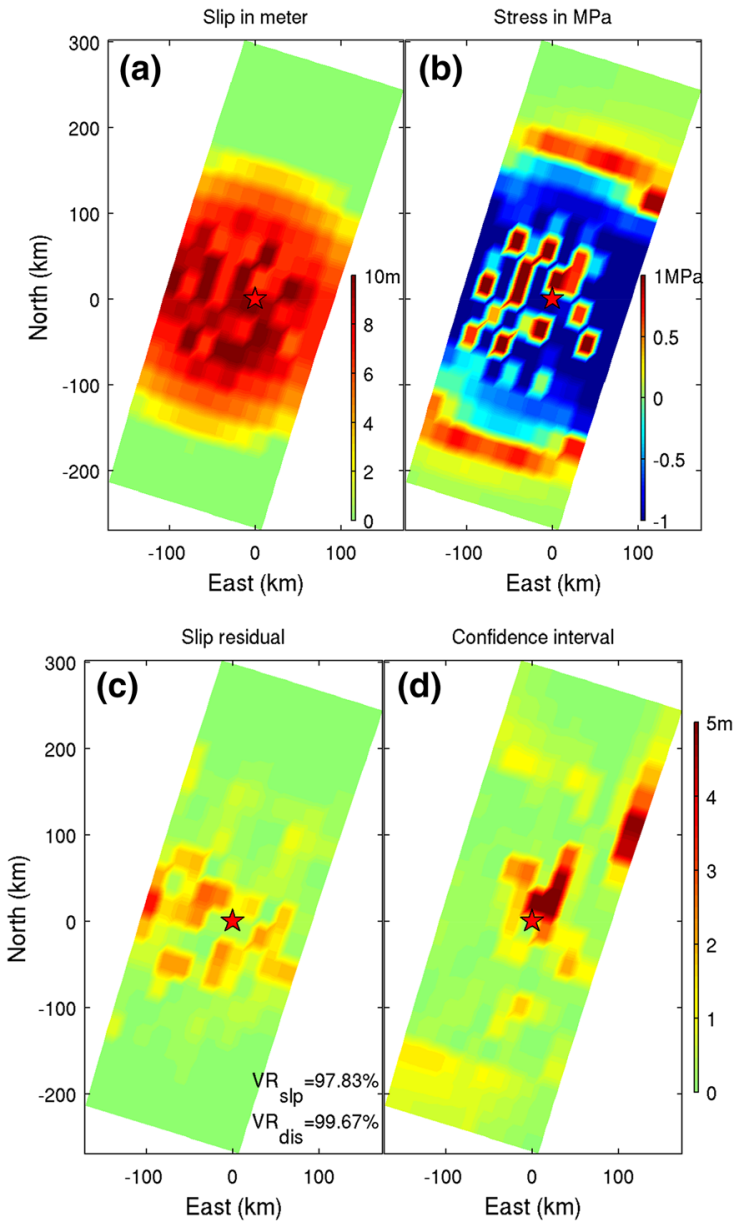

Figure 5

Color-coded slip model (a) used in Test VIII with its shear stress changes (b), the residual of the inverted optimal slip (c), and the slip uncertainty as the $95 \%$ credibility interval (d)

(marked as Test VIII). Such heterogeneity is likely reflected in the variance of the stress drop. The fault parameterization is the same as Test-0. Our slip model and its shear stress are shown in Fig. 5a and b, respectively. The inversion results, including the slip residuals (difference between the inverted optimal slip and the synthetic slip of Fig. 5a) and the uncertainties of slip estimation are shown in Fig. 5c and d, respectively. They indicate that the inverted slip with $\mathrm{VR}_{\text {slp }}$ of $97.8 \%\left(\mathrm{VR}_{\mathrm{dis}}\right.$ of $\left.99.91 \%\right)$ can retrieve the general feature of the input model, but the strong heterogeneity cannot be recovered. Meanwhile, the slip bias in the strongly heterogeneous zone is also shown to be inconsistent with the estimation 

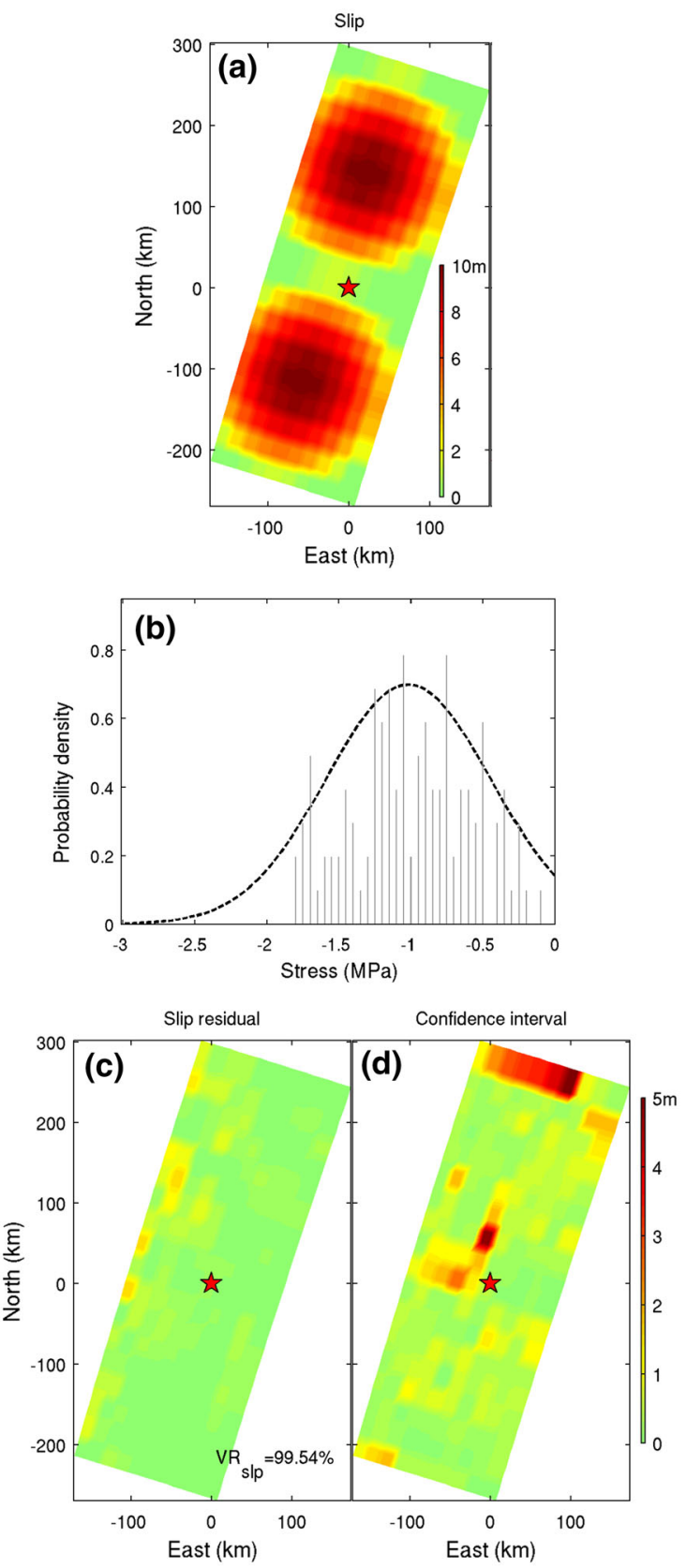

Figure 6

Color-coded slip model (a) used in Test IX and its corresponding probability density distribution of stress drops (b), where the dashed curve shows the inversion result and the gray bars refer to the true values. The residual slip distribution is displayed in panel (c) and the uncertainty of the estimated fault slip is shown in panel

(d)

uncertainty. Such a bias is mainly related to the applied prior PDF in the inversion, which imposes a weak smoothing condition (see Sect. 5.1). The inverted stress drop distribution is shown in Fig. 4 by the solid blue curve. As expected, when the slip is more heterogeneous, the stress drop has larger variance (Test-VIII vs. Test-0). Therefore, the variance of stress drop can reflect slip heterogeneity, but is also sensitive to fault parameterization, as indicated in Tests I-III and VI-VII.

In addition, we consider two slip clusters on the fault plane (Test IX). Shown in Fig. 6a, the input slip produces stress drop with mean of $-0.99 \mathrm{MPa}$ and standard deviation of $0.42 \mathrm{MPa}$ (see Fig. 6b). Based on the displacements produced, we invert for the fault slip and mean stress drop. In this inversion, we utilize a uniform PDF between [0.1 40] MPa for the mean stress drop to test the possible impact of the parameter range on the inversion results. The resulting slip residual between the estimated and the input slip (Fig. 6a) are provided in Fig. 6c and the uncertainty of slip estimation is given in Fig. 6d. The results indicate that the fault slip is well-retrieved with high $\mathrm{VR}_{\text {slp }}$ of $99.54 \%\left(\mathrm{VR}_{\text {dis }}\right.$ of $\left.99.96 \%\right)$ and the differences between the inverted and input slip are generally smaller than the estimation uncertainties. The reconstructed probability density of stress drop is shown in Fig. $6 \mathrm{~b}$ by the dashed curve. The estimated mean stress drop is $-1.02 \mathrm{MPa}$ and standard deviation is $0.32 \mathrm{MPa}$, which are consistent with the input model.

\section{Application to the 2010 M8.8 Maule Earthquake}

Since the 2010 M8.8 Maule earthquake was expected for decades, plentiful GPS measurements captured the displacements of the earthquake (VIGNY et al., 2011; MoRENO et al., 2012; LoRITO et al., 2011). Additionally, InSAR measured coseismic displacements, including 820 measuring points, were released by Tong et al. (2010). These geodetic data provide dense coseismic observations for the Maule earthquake.

Adopting the mechanically constrained inversion in the Bayesian approach, we invert for the stress drop and coseismic slip of the Maule earthquake, utilizing the fault geometry of USGS and LORITO et al. (2011), respectively. As described before, the rake angle is fixed as $110^{\circ}$. The matrix of Green's 

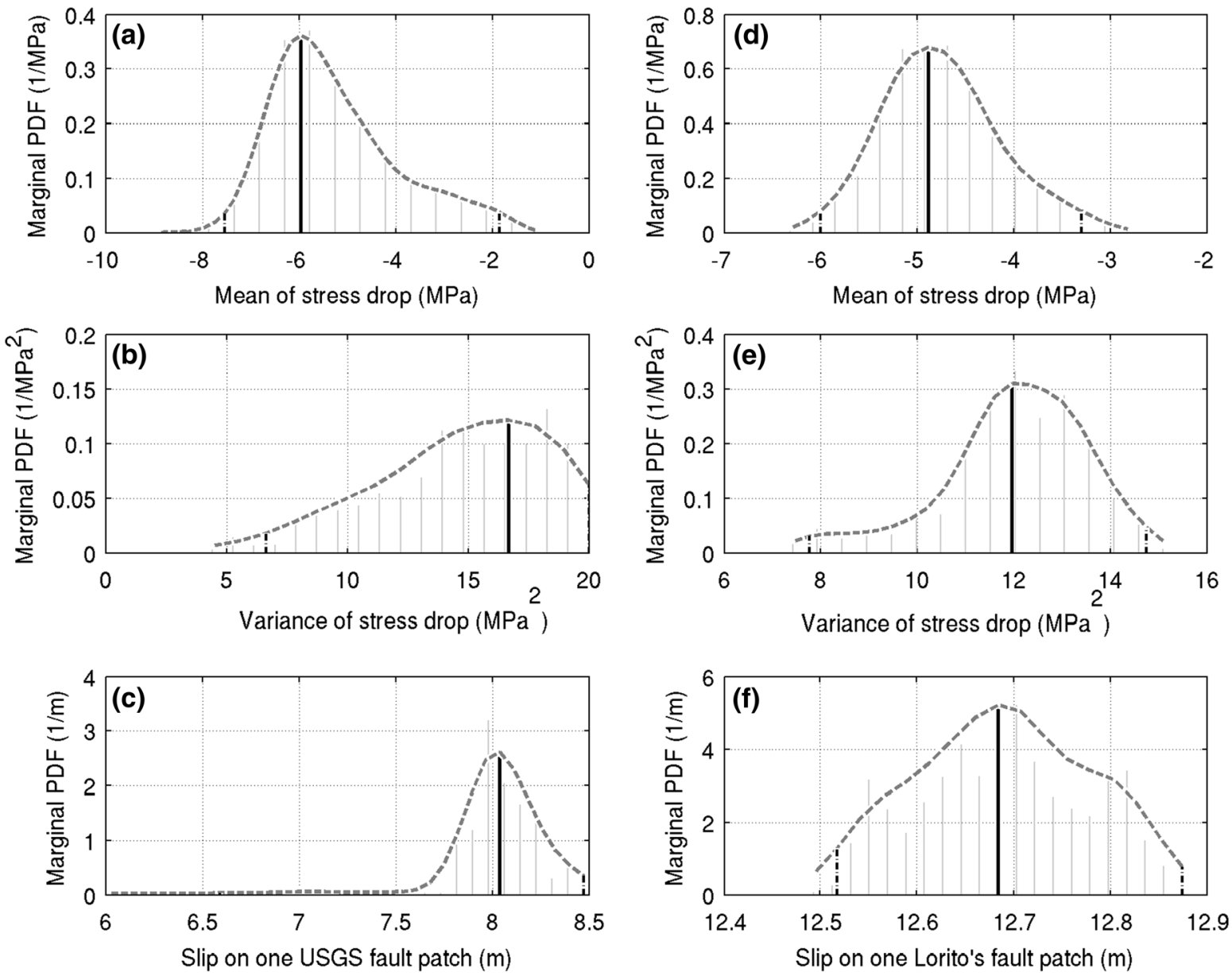

Figure 7

Marginal PDFs constructed from MCMC generated samples. Marginal PDFs of mean (a) and variance (b) of the stress drop estimation for the 2010 M8.8 Maule earthquake using the USGS fault plane; c marginal PDF of slip on the fault patch near the hypocenter ('+' marked in Fig. 8a, f) as an illustration. Panels (d-f) show the corresponding results for Lorito's fault geometry. The optimal values are marked by the solid black line and $95 \%$ credibility intervals are identified by the dashed black lines

function is constructed the same as that for the synthetic test in Sect. 3. We present the marginal PDFs based on the MCMC-generated samples for the mean and variance of stress drop in Fig. 7. Clearly peaked distributions are observed, and thus we may reliably select the value with maximum probability density as the representative optimal value of each parameter. The estimated stress drops are similar for both fault geometries, with mean/standard deviation of 6.0/ 4.3 MPa when USGS fault geometry is adopted, and mean/standard deviation of 5.0/3.4 MPa when Lorito's fault geometry is used. The distribution of the optimal slip is shown in Fig. 8. As an example, the marginal PDF for slip on one USGS fault patch near the hypocenter is displayed in Fig. 7c (Fig. 7f for Lorito's fault). The modeling results based either on the USGS or the Lorito's fault geometry explain the geodetic measurements well, with $\mathrm{VR}_{\text {dis }}$ of $99 \%$ for horizontal GPS measurements, $95 \%$ for InSAR data and higher than $80 \%$ for vertical GPS measurements. The relatively poorer data fit for the InSAR and the vertical GPS measurements is partly related to their large measurement uncertainties and thus lower weighting during the inversion (see Eq. 1). The results show that the general slip pattern is similar for both fault geometries, indicating a major slip 

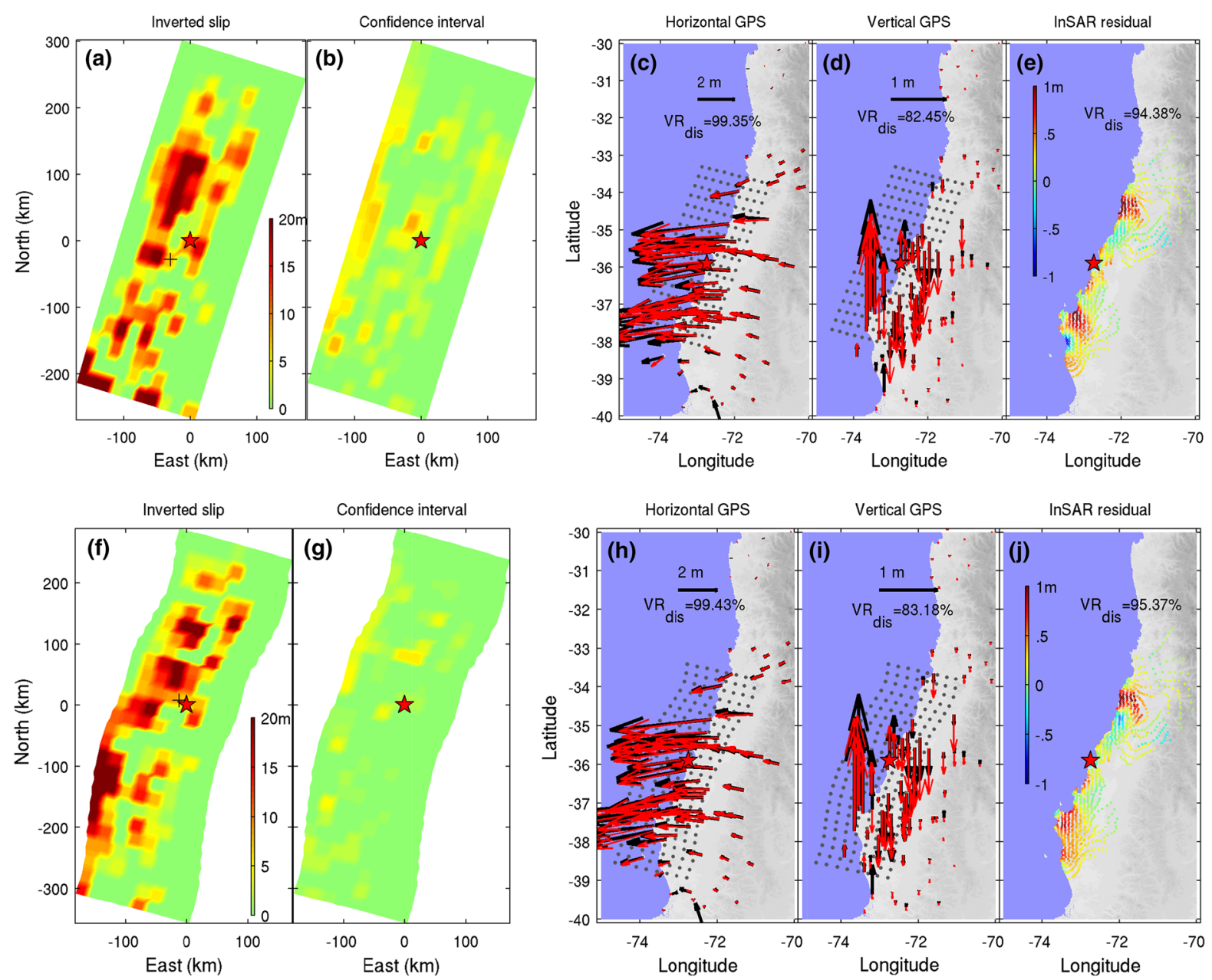

Figure 8

Color-coded slip model for the 2010 Maule earthquake using USGS fault geometry (a) and its uncertainty (b). Color-coded slip model using Lorito's fault geometry (f) and its uncertainty (g). The corresponding displacements are shown in panels $\mathbf{c}-\mathbf{e}$ and $\mathbf{h}-\mathbf{j}$. The observed and modeled GPS displacements are displayed by black and red arrows, respectively. The star indicates the location of the Maule earthquake. In panel (a) and (f), '+' marks the patch on which the sampled slip is presented in Fig. 7c, f

concentration north of the hypocenter. This is basically consistent with other published modeling results (e.g., Lorito et al., 2011; Vigny et al., 2011; Tong et al., 2010; Moreno et al., 2012). In more detail, our derived slip model based on Lorito's fault geometry (Fig. 8f) provides shallower slip south of the Maule hypocenter than that based on the USGS fault plane, indicating that slip inversion is sensitive to the utilized fault geometry. In comparison with the slip model of LoRITo et al. (2011), our derived shallow slip can be explained by the fact that we do not include any constraint such as zero-slip constraint along the upper border (LORITO et al., 2011).
In addition, we test inversion based only on the GPS measurements. The results for USGS and Lorito's fault are provided in the supplementary material (Fig. S9, S10). The inverted fault slip has a similar pattern as that shown in Fig. 8 and the stress drop has a mean of 6.6/5.3 MPa and a standard deviation of 4.1/3.1 MPa for the USGS/Lorito's fault plane, respectively. In addition, we test a different fault discretization to the USGS fault plane with $26 \times 11$ patches. The inverted coseismic slip for the Maule earthquake is displayed in Fig. S11 of the supplementary material. The inverted stress drop has mean of 5.9 $\mathrm{MPa}$ and standard deviation of $4.8 \mathrm{MPa}$. The 
inversion results are basically consistent with those using entire GPS and InSAR dataset, indicating the stability of the inversion approach.

\section{Discussion}

\subsection{Dependency of Stress Drop on Smoothing Conditions in the Traditional LS Slip Inversion}

Stress drop is a key parameter in earthquake mechanics. The relation of the stress drop to other parameters, e.g., earthquake magnitude, is a matter of debate. For example, the stress drop of the 2011 M9 Tohoku-Oki event, which caused a disastrous tsunami, was suggested to be four times higher than that of the 2010 M8.8 Maule, Chile, earthquake (Simons et al., 2011; Vigny et al., 2011; Delouis et al., 2010). To constrain the dependence of stress drop on other parameters, it is important to get precise estimates of stress drop from independent data. However, depending on the measuring approach, stress drop can be considerably biased due to uncertainties in geological measurements, in the source spectrum or in the fault slip model. In particular, the approaches based on geological measurements and the source spectrum assume a circular rupture or a uniform rectangular dislocation, which is unrealistic for a large earthquake with usually complex rupture.

For large earthquake ruptures, it is convenient to estimate stress drop from geodetically (or seismically) determined slip distributions. Nevertheless, fault slip based on the traditional least-square (LS) approach is usually not unique and depends strongly on the specified smoothing condition. For example, when the Laplacian filter $(\mathbf{L})$ is applied in the inversion, the slip is determined according to $\mathbf{b}\left(\beta^{2}\right)=$ $\arg \min \left(\|\mathbf{G b}-\mathbf{y}\|^{2}+\beta^{2}\|\mathbf{L b}\|^{2}\right)$, with $\beta^{2}$ being the smobthing parameter. Larger $\beta^{2}$ means stronger smoothing imposed on the slip model at the expense of poorer data fitting. In Fig. 9, we demonstrate the tradeoff curve between data fitting (Root-meansquare value) and the smoothing condition for the Maule earthquake based on the LS approach. In the LS inversion, the final smoothing factor is usually selected at the point of strong curvature of the RMS curve in order to balance data fitting and smoothing.
However, as observed in Fig. 9, the stress drop that is calculated as the mean value of negative shear stress on the fault plane, changes from 2 to $6 \mathrm{MPa}$ with the applied smoothing factor. In particular, significant changes occur when data fitting and smoothing is usually thought to be appropriately balanced (at the strong curvature). Thus, the final stress drop value strongly depends on the arbitrarily chosen smoothing factor.

As mentioned before, our mechanical constraint introduced as the prior information in the inversion also imposes a loose smoothing condition on the fault slip. For demonstration, we calculate $\mathbf{b}^{\mathrm{T}} \mathbf{L}^{\mathrm{T}} \mathbf{L b}$ (a measure of the roughness of the slip distribution), with slip b being taken from the MCMC-generated samples in Test-0. In Fig. 10, the values of $\mathbf{b}^{\mathrm{T}} \mathbf{L}^{\mathrm{T}} \mathbf{L b}$ are plotted against the sampled variance of the stress drop. It indicates that the slip roughness is positively correlated with the variance of stress drop, showing that smaller variance corresponds to a smoother slip distribution. Therefore, the mechanical constraint plays a similar role as the Laplacian smoothing imposed in the traditional LS inversion. However, the slip inversion with mechanical constraints provides additional stress drop information about the earthquake without the subjective setting of a smoothing parameter as in the LS approach. In those cases where stress drop information is available from other data/ approaches, such information can be incorporated into the inversion as a constraint on fault slip.

\subsection{Statistical Distribution of Stress Drop}

Statistical distributions for stress drop generally involve intra-event and inter-event variability, respectively. The former considers shear stress variability on the fault for one earthquake rupture, and its knowledge is important for dynamic simulation and ground-motion prediction; the latter delineates the variability of the mean stress drop from different earthquakes on a global or regional scale. It has been reported that the stress drop from finite-fault slip inversions can be described as random fields modeled by a von Karman auto-correlation function (MAI and BEROZA, 2002), and its power spectrum in the wavenumber space is characterized by a power law decay. Fractal distributions have the advantage of 


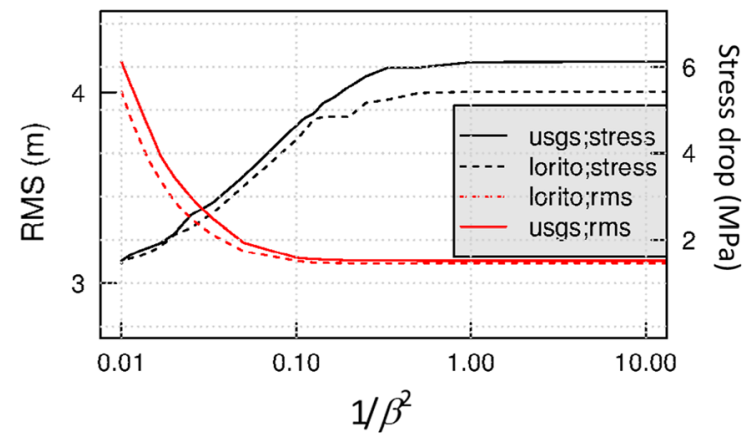

Figure 9

Root-mean-square (RMS) errors of the data fits based on the LS approach (red solid curve for the USGS fault plane; red dashed curve for Lorito's fault plane) and the corresponding mean stress drop values (mean values of negative shear stress due to slip on the fault plane) as a function of the smoothing factor

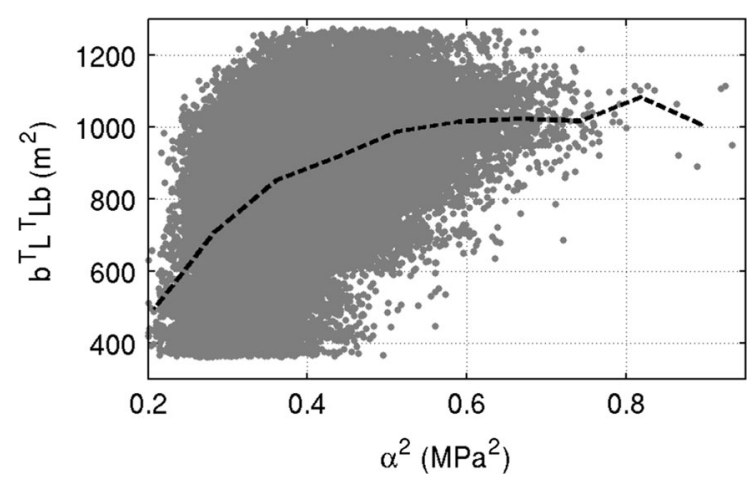

FigureFig. 10

Slip roughness $\left(\mathbf{b}^{\mathrm{T}} \mathbf{L}^{\mathrm{T}} \mathbf{L b}\right)$ is plotted against the variance of stress drop during the MCMC sampling of the Test- 0 . The dashed curve indicates the median values of $\mathbf{b}^{\mathrm{T}} \mathbf{L}^{\mathrm{T}} \mathbf{L b}$ for different $\alpha^{2}$

delineating the short-scale variation and are particularly important in the simulation of high frequency wave propagation in the near field in dynamic modeling, while the Gaussian distribution and von Karman auto-correlation function provide similar fits for a larger ( $>1 \mathrm{~km}$ ) spatial scale (MAI and BEROZA, 2002). Because our approach is based on static surface displacements, and thus ignores the effects of high frequencies, the Gaussian distribution can be seen as an appropriate choice of statistical distribution of stress drop. On the other hand, the lognormal distribution has been proposed for describing regional/global inter-event variability (e.g., ANDREws, 1986; Allmann and Shearer, 2007). With a simple assumption that the large rupture is composed of numerous sub-events with different sizes, we have also tested to implement the lognormal instead of the Gaussian distribution as a prior in our inversion approach. However, since the difference in the logarithmic stress drop is small for different slip models, the MCMC sampling process did not sufficiently converge in this case.

Thus, we utilize the Gaussian distribution as an approximation of the stress drop variability. Stress drop delineated as uncorrelated random numbers around its central value has also been applied in quasi-dynamic modeling (e.g., Ben-Zion, 1996).

\section{Conclusion}

Slip distributions inferred from seismologic and geodetic data provide a valuable constraint to estimations of stress drop. However, the impact of the errors in the slip model on the stress drop estimation is not at all clear. In our approach, slip and stress drop are included in the same statistical model and can be estimated simultaneously, which allows for a straightforward probabilistic uncertainty handling. By implementing a mechanical constraint, the explicit smoothing prior is eliminated and stress drop can be determined together with slip on the fault. Our synthetic tests indicate that the inversion is stable, and reliably retrieves the major features of both slip and stress drop. We apply the inversion approach to the 2010 M8.8 Maule earthquake. The results show that the slip distributions are rougher but generally similar to those obtained in the previous studies. Furthermore, the mean stress drop obtained is close to the value independently calculated from the force balance approach (LuTtRell et al., 2011). Thus, our technique could serve as a tool to study large earthquakes in terms of slip and stress drop by replacing the arbitrary smoothing condition with a physical constraint. The resulting consistencies in the synthetic tests and the reliable estimations of slip and stress drop for the Maule event, indicate that the mechanically constrained inversion can be potentially applied to the quantitative evaluations of stress drop during large ruptures and of stress accumulation rate during the interseismic phase, which further serves 
for evaluating recurrence intervals of large earthquakes.

\section{Acknowledgments}

We would like to thank the editor and the anonymous reviewers for their very detailed and constructive reviews. Furthermore, we acknowledge Yehuda BenZion for stimulating discussions. This work was supported by NSFC-41204065 and BMBF-PROGRESS project.

Open Access This article is distributed under the terms of the Creative Commons Attribution License which permits any use, distribution, and reproduction in any medium, provided the original author(s) and the source are credited.

\section{REFERENCES}

Allmann, B. P., and P. M. Shearer (2007), Spatial and temporal stress drop variations in small earthquakes near Parkfield, California, J. Geophys. Res., 112(B4), 1-17.

ANDREWs, D. J. (1974), Evaluation of static stress on a fault plane from a Green's function, Bull. Seis. Soc. Am, 64(6), 1629-1633.

Andrews, D. J. (1980), A stochastic fault model. 1. Static case, J. Geophys. Res., 85, 3867-3877.

ANDREws, D. J. (1986), Objective determination of source parameters and similarity of earthquakes of different size, in Earthquake Source Mechanics, edited by S. Das, J. Boatwright and C. H. Scholz, pp. 259-267, American Geophysical Union, Washington, DC.

AtKinson, G. M., and I. Beresnev (1997), Don't call it stress drop, Seism. Res. Lett., 68(1), 3-4.

Bayes, T., and R. Price (Eds.) (1763), An essay towards solving a problem in the Doctrine of chance. By the late Rev. Mr. Bayes, communicated by Mr. Price, in a letter to John Canton, M. A. and F. R. S., 370-418 pp., Philosophical Transactions of the Royal Society of London.

BECK, J. L. (2010), Bayesian system identification based on probability logic, Struct. Contrl. Health. Monit., 17, 825-847.

BECK, J. L., and L. KATAFYGIOTIS (1998), Updating models and their uncertainties, Part I: Bayesian statistical framework, J. Eng. Mech, 124(4), 455-461.

Becker, T. W., J. L. Hardebeck, and G. Anderson (2005), Constraints on fault slip rates of the southern California plate boundary from GPS velocity and stress inversions, Geophys. J. Int., 160(2), 634-650.

Ben-Zion, Y. (1996), Stress, slip, and earthquakes in models of complex single-fault systems incorporating brittle and creep deformations, J. Geophys. Res., 101, 5677-5706.

Bouchon, M., M. Campillo, and F. Cotton (1998), Stress field associated with the rupture of the 1992 Landers, California, earthquake and its implications concerning the fault strength at the onset of the earthquake, Journal of Geophysical Research, 103(B9), 21091-21097.

Brune, J. N. (1970), Tectonic stress and the spectra of seismic shear waves from earthquakes, J. Geophys. Res., 75(26), 4997-5009.

Cotton, F., R. Archuleta, and M. Causse (2013), What is sigma of the stress drop?, Seism. Res. Lett., 84(1), 42-48.

Daston, L. (Ed.) (1988), Classical Probability in the Enlightenment, Princeton Univ Press.

Delouis, B., J.-M. Nocquet, and M. Vallee (2010), Slip distribution of the Feburary 27, $2010 \mathrm{Mw}=8.8$ Maule Earthquake, central Chile, from static and high-rate GPS, InSAR, and broadband teleseismic data, Geophys. Res. Lett., 37(17).

Du, Y., A. Aydin, and P. Segall (1992), Comparison of various inversion techniques as applied to the determination of a geophysical deformation model for the 1983 Borah Peak earthquake, Bull. Seis. Soc. Am., 82(4), 1840-1866.

Fukao, Y., and M. Furumoto (1978), Stress drops, wave spectura and recurrence intervals of great earthquakes-implications of the Etorofu earthquake of 1958 November 6, Geophys. J. Int, 57(1), 23-40.

FukUdA, J., and K. M. Johnson (2008), A fully Bayesian inversion for spatial distribution of fault slip with objective smoothing, Bull. Seism. Soc. Am., 98(3), 1128-1146.

Gelfand, A. E., and A. F. M. Sмith (1990), Sampling-based approaches to calculating marginal densities, J. Am. Stat. Asso., 85, 398-409.

Guatteri, M., P. M. Mai, and G. C. Beroza (2004), A pseudodynamic approximation to dynamic rupture models for strong ground motion prediction, Bull. Seism. Soc. Am., 94(6), 2051-2063.

HANKs, T. C., and R. K. McGuIRE (1981), The character of highfrequency strong ground motion, Bull. Seism. Soc. Am., 71, 2071-2095.

Hartzell, S. H., and D. V. Helmberger (1982), Strong-motion modeling of the Imperial Valley earthquake of 1979, Bull. Seism. Soc. Am., 72, 571-596.

Hastings, W. K. (1970), Monte Carlo Sampling methods using Markov Chains and their applications, Biometrika, 57(1), 97-109.

Johnson, L. R. (1979), Seismic source theory, Rev. Geophys. Space Phys., 17, 328-336.

KANAmori, H. (1994), Mechanics of earthquakes, Ann. Rev. Earth Planet. Sci., 22, 207-237.

Kanamori, H., and D. L. Anderson (1975), Theoretical basis of some empirical relations in seismology, Bull. Seism. Soc. Am., 65, 1073-1096.

Косн, K.-R. (2007), Introduction to Bayesian Statistics, 2 ed.

Lay, T., C. J. Ammon, H. Kanamori, K. D. Koper, O. Sufri, and A. R. Нuтко (2010), Teleseismic inversion for rupture process of the 27 February 2010 Chile (Mw 8.8) earthquake, Geophys. Res. Lett., 37(13).

Lohman, R., and M. Simons (2005), Some thoughts on the use of InSAR data to constrain models of surface deformation: Noise structure and data downsampling, Geocham. Geophys. Geosyst., 6(1).

Lorito, S., F. Romano, S. Atzori, X. Tong, A. Avallone, J. McCloskey, M. Cocco, E. Boschi, and A. Piatanesi (2011), Limited overlap between the seismic gap and coseismic slip of the great 2010 Chile earthquake, Nature. 
Luttrell, K., X. Tong, D. Sandwell, B. Brooks, and M. Bevis (2011), Estimates of stress drop and crustal tecnic stress from the 27 February 2010 Maule, Chile, earthquake: Implications for fault strength, J. Geophys. Res.

Madariaga, R. (1976), Dynamics of an expanding circular fault, Bull. Seism. Soc. Am., 66, 639-666.

MAI, P. M., and G. C. BEROZA (2000), Source scaling properties from finite-fault-rupture models, Bull. Seism. Soc. Am., 90(3), 604-615.

MaI, P. M., and G. C. Beroza (2002), A spatial random field model to characterize complexity in earthquake slip J. Geophys. Res., 107(B11,2308).

Metropolis, N., A. W. Rosenbluth, M. N. Rosenbluth, A. H. Teller, and E. Teller (1953), Equations of state calculations by fast computing machines, Journal of Chemical Physics, 21(6), 1087-1092.

Minson, S. E., M. Simons, and J. L. BECK (2013), Bayesian inversion for finite fault earthquake source models I - theory and algorithm, Geophys. J. Int., 194(3), 1701-1726, doi:10.1093/gji/ ggt180.

Monelli, D., and P. M. MAI (2008), Bayesian inference of kinematic earthquake rupture parameters through fitting of strong motion data, Geophys. J. Int, 173(1), 220-232.

Monelli, D., P. M. Mai, S. Jónsson, and D. Giardini (2009), Bayesian imaging of the 2000 Western Tottori (Japan) earthquake through fitting of strong motion and GPS data, Geophys. J. Int., 176(1), 135-150.

Moreno, M., J. Klotz, D. Melnick, H. Echtler, and K. Bataille (2008), Active faulting and heterogeneous deformation across a megathrust segment boundary from GPS data, south central Chile (36-39 ${ }^{\circ}$ ), Geochem. Geophys. Geosyst., 9(12), 1-14.

Moreno, M., et al. (2012), Toward understanding tectonic control on the Mw 8.82010 Maule Chile earthquake, Earth and Planetary Science Letters, 152-165.

ОкарА, Y. (1992), Internal deformation due to shear and tensile faults in a half-space, Bull. Seism. Soc. Am., 82, 1018-1040.

Page, M., S. Custodio, R. Archuleta, and J. M. Carlson (2009), Constraining earthquake source inversions with GPS data: 1. Resolution-based removal of artifacts, J. Geophys. Res., 114(B01314).

PARsons, T. (2006), $M \geq 7.0$ earthquake recurrence on the San Andreas fault from a stress renewal model, J. Geophys. Res., 111(B12), 1-15.

Pollitz, F., et al. (2011), Coseismic slip distribution of the Feburary 27, 2010 Mw8.8 Maule, Chile earthquake, Geophys. Res. Lett., 38(9).
Ripperger, J., and P. M. Mai (2004), Fast computation of static stress changes on $2 D$ faults from final slip distributions, Geophys. Res. Lett., 31(18).

Ruegg, J. C., J. Campos, R. Madariaga, E. Kausel, J. B. de Chabalier, R. Armijo, D. Dimitrov, I. Georgiev, and S. BARRIENTOS (2002), Interseismic strain accumulation in south central Chile from GPS measurements, 1996-1999, Geophys. Res. Lett., 29(11), 12-11 to 12-14.

Ruegg, J. C., A. Rudloff, C. Vigny, R. Madariaga, J. B. Chabalier, J. Campos, E. Kausel, S. Barrientos, and D. Dimitrov (2009), Interseismic strain accumulation measured by GPS in the seismic gap between Constitucion and concepcion in Chile, Physics of the Earth and Planetary Interiors, 175, 78-85.

RuFF, L. J. (1999), Dynamic stress drop of recent earthquakes: variations within subduction zones, Pageoph, 154, 409-431.

Shearer, P. M., G. A. Prieto, and E. Hauksson (2006), Comprehensive analysis of earthquake source spectra in southern California, J. Geophys. Res., 111(B06303).

Simons, M., et al. (2011), The 2011 Magnitude 9.0 Tohoku-Oki Earthquake: Mosaicking the Megathrust from Seconds to Centuries, Science, 332(6036), 1421-1425.

SNieder, R., and J. TRAmpert (Eds.) (1999), Inverse problems in geophysics Springer Verlag, New York.

Sun, J., K. M. Johnson, Z. Cao, Z. Shen, R. Burgmann, and X. Xu (2011), Mechanical constrains on inversion of coseismic geodetic data for fault slip and geometry: Example from InSAR observation of the 6 October 2008 Mw6.3 Dangxiong-Yangyi (Tibet) earthquake, J. Geophys. Res., 116(B01406).

Tarantola, A. (2005), Inverse problem theory and methods for model parameter estimation, SIAM, Philadelphia, PA, US.

Tong, X., et al. (2010), The 2010 Maule, Chile earthquake: downdip ruptrue limit revealed by space geodesy, Geophys. Res. Lett., 37.

VIGNY, C., et al. (2011), The 2010 Mw 8.8 Maule mega-thrust earthquake of Central Chile, monitered by GPS, Science, 332, 1417-1421.

Wang, L., S. Hainzl, G. Zoeller, and M. Holschneider (2012), Stress- and aftershock- constrained joint inversions for co- and post- seismic slip applied to the 2004 M6.0 Parkfield earthquake, J. Geophys. Res.

WANG, L., S. HAInzl, and G. Zoller (2014), Assessment of stress coupling among the inter-, co- and post-seismic phases related to the 2004M6 Parkfield earthquake, Geophys. J. Int.

YABUKI, T., and M. MATSU'URA (1992), Geodetic data inversion using a Bayesian information criterion for spatial distribution of fault slip, Geophys. J. Int, 109, 363-375. 